\title{
A importância das universidades e institutos de pesquisa para o sistema de inovação da Região Norte
}

\author{
Leandro Morais de Almeida ${ }^{1}$ \\ Márcia Juca Teixeira Diniz ${ }^{2}$ \\ Ana Paula Bastos ${ }^{3}$ \\ Marcelo Bentes Diniz ${ }^{4}$ \\ Lívia Maria de Araújo Cavalcante ${ }^{5}$
}

\begin{abstract}
Resumo: Este artigo analisa a importância das universidades e institutos públicos de pesquisa para o Sistema de Inovação da Região Norte. A pesquisa parte da base de dados elaborada com grupos de pesquisa cadastrados no Conselho Nacional de Desenvolvimento Científico e Tecnológico (CNPq), que declararam algum tipo de relacionamento com empresas do setor produtivo. Pesquisas internacionais, Carnegie Mellon Survey e o Yale Survey, bem como nacionais, Minas Gerais Survey, apontam como cruciais para o desenvolvimento, as interações entre essas instituições e as empresas. Os resultados sugerem que as universidades apresentam importante papel complementar e até mesmo substituto de Pesquisa e Desenvolvimento da indústria da região, apesar da demanda deste conhecimento está aquém da capacidade inovadora das universidades, em função do incipiente investimento em ciência e tecnologia.
\end{abstract}

Palavras-chave: sistema regional de inovação; universidade; empresa.

JEL: O14; O25; O33.

\footnotetext{
1 Doutorando pelo Programa de Pós Graduação em Desenvolvimento Sustentável do Trópico Úmido da Universidade Federal do Pará (UFPA). E-mail: lmorais@ufpa.br

2 Professora do Programa de Pós Graduação em Economia da Universidade Federal do Pará (PPGE/UFPA).

E-mail: mjucadiniz@hotmail.com

3 Professora do Programa de Pós Graduação em Economia da Universidade Federal do Pará (PPGE/UFPA).

E-mail: pbastos@ufpa.br

4 Professor do Programa de Pós Graduação em Economia da Universidade Federal do Pará (PPGE/UFPA).

E-mail: mbdiniz@ufpa.br

5 Bacharel em Ciências Econômicas pela UFPA. E-mail: liviaeconomista@yahoo.com.br
} 


\title{
The importance of universities and research institutes to the innovation system in the northern region
}

\begin{abstract}
This paper analyzes the importance of universities and public research institutes for the Innovation System of the North Region. The sample was taken from a database of research groups registered in National Agency of Research Funding, CNPq that declared some kind of innovative relationship with firms. Research International, Carnegie Mellon Survey and Yale Survey, as well as national, Minas Gerais survey, as a crucial point for the development, interactions between these institutions and firms. Results suggest that universities play a key role to complement and even substitute for $R \& D$ industry of the region, despite the demands of this knowledge is far behind the innovative capacity of universities, according to the incipient investment in science and technology.
\end{abstract}

Key-words: regional system of innovation; university; firms.

JEL: O14; O25; O33.

\section{Introdução}

O Sistema Regional de Inovação (SRI) é um arranjo institucional envolvendo múltiplos agentes (empresas; universidades e institutos de pesquisa; instituições de ensino em geral; sistema financeiro; sistemas legais; mecanismos mercantis; e governos), que não estão unicamente aglomerados em uma determinada localização mas que interagem gerando conhecimento partindo da base local mas com ligações nacionais e globais (Asheim; Coenen, 2005). A base destes sistemas de inovação se constrói através dos setores produtivos e, as empresas que saem na frente (como uma típica empresa schumpeteriana), incorporam continuamente conhecimento tácito, estratégias direcionada a inovação, know how, baseada em atividades de rotina (Nelson; Winter, 1982).

Em face do atual cenário de globalização e da rapidez com que se desenvolvem e difundem as inovações tecnológicas é fundamental que universidades e instituições de pesquisa, criem potencial para o desenvolvimento de ciência e tecnologia dando suporte ao setor produtivo, onde, em países em desenvolvimento, este setor apresenta baixos investimentos para este fim. As relações entre universidades e institutos de pesquisas e empresas, juntamente com todos os agentes sociais de uma economia devem constituir um sistema de inovação pautado ao atendimento às necessidades prioritárias do desenvolvimento sócio-econômico. 
A região Norte possui universidades e instituições importantes para a pesquisa científica que podem vir a contribuir para o desenvolvimento de áreas como a biotecnologia, a qual tem na riqueza de sua flora e fauna um potencial único no mundo. Entretanto, o sistema de inovação da região encontra-se ainda em um estágio de formação onde as bases para a ocorrência de processos interativos entre universidades empresas são fracamente constituídas (Puty et al., 2008).

Desse modo, o presente artigo, tem por objetivo, analisar os padrões de interação entre universidades e institutos públicos de pesquisa com empresas na região Norte, visando compreende a contribuição das instituições acadêmicas para o Sistema de Inovação da região. Para tanto, o artigo conta com quatro seções, além desta introdução. A segunda seção apresenta uma revisão bibliográfica sobre o desenvolvimento e os sistemas nacionais e regionais de inovação. O terceiro apresenta a base produtiva da região e os indicadores de Ciência e Tecnologia. O quarto descreve a metodologia utilizada no trabalho de pesquisa, e no quinto são discutidos os resultados da pesquisa, onde é realizada uma análise mais detalhada da interatividade entre universidades/instituições e empresas nos estados da região Norte.

\section{O desenvolvimento na perspectiva da inovação}

O referencial analítico deste trabalho privilegia a abordagem interativa do processo de inovação, presente na Teoria Neo-Schumpteriana/ Teoria Evolucionária. Segundo essa Teoria as empresas não inovam isoladamente, mas constantemente o fazem num contexto de redes de relações diretas ou indiretas, com outras empresas, instituições de ensino e pesquisa, infraestrutura de pesquisa pública e privada, economia nacional e internacional, entre outros.

Existem, três fases básicas para o processo de inovação sintetizada como: a invenção, como resultado de um processo de descoberta, de princípios técnicos novos, potencialmente abertos para exploração comercial, mas não necessariamente realizada; a inovação, como o processo de desenvolvimento de uma invenção de forma comercial; a difusão, como a expansão de uma inovação em uso comercial, novos produtos e processos (Nelson; Winter, 1982; Dosi, 2006, Freeman, 1990)

O pensamento neo-schumpeteriano analisa o emprego da inovação na evolução e configuração das estruturas industriais. Trata a introdução e difusão de inovações como um processo que resulta no crescimento diferenciado por parte das empresas. A dinâmica do mercado ocorre mediante um processo de seleção natural, ou seja, os produtores que não conseguem acompanhar a velocidade e as direções do progresso técnico são expulsos ou incorporados 
pelos concorrentes. Neste contexto, as empresas para sobreviverem precisam adaptar-se continuamente as novas condições do mercado, em função das inovações tecnológicas adotadas por outras empresas (Nelson; Winter, 1982).

A Teoria Evolucionária forma, por assim dizer, a base do conceito sobre Sistemas Nacionais de Inovação, campo de estudo teórico que aborda a inovação, a composição de ciência e tecnologia, os agentes que os compõem e conseqüentemente o desenvolvimento de um país ou região.

\subsection{Os sistemas nacionais e regionais de inovação}

A análise dos Sistemas Nacionais de Inovação (SNI), do qual se deriva o conceito de Sistemas Regionais de Inovação (SRI) permite compreender os processos de criação, uso, e difusão do conhecimento. Procura evidenciar o processo de inovação, a importância da interação entre os diversos agentes como: instituições, empresas, governo, ONGs e outros, inseridos numa determinada região. Esse sistema se baseia nas principais características do atual processo de produção e de acumulação, quais sejam: criatividade, inovações radicais e incrementais, difusão, e a importância da localização geográfica na geração de inovações. (Freeman, 1990).

Um aspecto importante é a natureza sistêmica para o processo de inovação, em especial, para países em desenvolvimento. Os fatores sistêmicos funcionam como estratégia de crescimento para alcançar um melhor desempenho tecnológico e conseqüentemente o desenvolvimento econômico de um determinado país ou região.

Uma estratégia de desenvolvimento (sistêmica) baseada no SRI tem como ponto de partida, uma analise de todos os setores da economia que contribuem para o desenvolvimento de competências visando a inovação. Essa análise pode identificar as redes de estimulo ao aprendizado e as redes e interações ausentes. $\mathrm{O}$ sistema nacional de inovação tem como pressuposto uma interação acentuada entre a ciência pura (Rosenberg, 2006) e a tecnologia, mostrando que as empresas obtêm melhores resultados à medida que a pesquisa básica progride.

Os mecanismos de aprendizado são importantes e podem ser tanto interno como externo à empresa (Dosi, 1984). A atenção às fontes internas vai exigir uma aproximação entre as fases de criação, e, portanto, superar a falta de comunicação entre as etapas do processo de inovação.

A constituição da capacidade tecnológica, ao nível da empresa dependerá também - mecanismos externos - da capacidade de interação das empresas com seus clientes, competidores, contratadores, universidades, laboratórios, 
agentes governamentais, consultores e pesquisadores (Freeman, 1990), e os formatos dessa capacidade podem ser os mais diversos desde a engenharia reversa até a colaboração informal.

Suzigan e Albuquerque (2006) destacam as principais contribuições das instituições cientificas em um SRI imaturo: instrumento de focalização contribuindo para a identificação de oportunidades e de vinculação do país aos fluxos internacionais; apoio para o desenvolvimento industrial, fornecendo o conhecimento necessário para a entrada em setores industriais estratégicos; e, como fonte para algumas soluções criativas que dificilmente seriam obtidas fora do país.

Mais especificamente, a contribuição da pesquisa acadêmica para o avanço tecnológico ocorre por vários mecanismos, além de variar entre os setores industriais e de existirem ramos da ciência cujos avanços são considerados mais relevantes para as inovações tecnológicas. Klevorick (1995) aponta os avanços no conhecimento científico como sendo a fonte mais importante de oportunidades tecnológicas.

As universidades produzem ciência para dar suporte à atividade inovativa das empresas (Klevorick, 1995; Cohen, 2002). É importante ressaltar que a interação universidade-empresa é específica a cada país e depende da infra-estrutura nacional de Ciência e Tecnologia (C\&T).

Por essa interação pode se abrir um leque de oportunidades de trabalho para todos os agentes envolvidos - alunos, pesquisadores, entre outros, e assim, uma coincidência de interesses entre o setor produtivo e a academia. A prática é fundamental para estimular temas de pesquisa, ao menos à pesquisa com fins aplicativos.

Como bem demonstram Cassiolato e Lastres (1998), os investimentos em P\&D e capacitação técnica para estimular a inovação e a difusão de tecnologias, além de inovações organizacionais e institucionais, podem conduzir a economia para um crescimento sustentado. Assim, cabe aos países e regiões criar um ambiente institucional capaz de propiciar a capacitação técnica, a inovação, a difusão e a incorporação de novas tecnologias. Este ambiente ou arranjo institucional deverá favorecer: o relacionamento/interdependência entre pesquisa básica e pesquisa aplicada, entre os pesquisadores e empresários, a inovação, a difusão e a incorporação das novas tecnologias dentro e fora dos limites regionais, e a crescente qualificação da mão de obra. 


\subsection{Breve histórico das políticas industriais e tecnológicas brasileiras}

O PDN voltado para o MSI que vigorou desde a década de 30 até o início dos anos 8o, foi capaz de construir um parque industrial complexo com elevados níveis de integração interssetorial. Entretanto, apresentava insuficiente desenvolvimento tecnológico, deficiências técnicas e específicas e principalmente, possuía pouca integração com o mercado internacional.

Nesse período o padrão de investimento e de instalação de setores industriais brasileiros foi baseado na atração generalizada de multinacionais, ocorrendo assim, um paradoxo: uma indústria com forte presença de capital estrangeiro, mas extremamente fechada, com quase nenhuma inserção internacional. Segundo Carleal (1997), a forte presença de empresas multinacionais trouxe como consequência à atividade de pesquisa básica desenvolvida nas matrizes dessas empresas e, portanto, fora do país.

Durante a década de 1980 a perda de dinamismo da indústria brasileira estava atrelada à limitada capacidade para desenvolver novas tecnologias com novos produtos, novos processos de produção dentro de uma organização mais eficiente e mais enxuta (Coutinho, 1994).

A partir da década de 1990, se inicia um intenso processo de reformas econômicas e institucionais destinadas à retomada do processo de desenvolvimento brasileiro, só que agora, num contexto de internacionalização, emergência de novos padrões de competição e aceleração do progresso técnico, ou melhor, dentro do novo padrão de expansão da economia mundial (Diniz, 2008).

Em meados da década de 1990, o Governo Federal apresenta a Nova Política Industrial e de Comércio Exterior (PICE). Para tal, o Estado criaria condições macroeconômicas, infra-estruturais e institucionais, além de exibir programas, tais como o Programa de Produtividade e Qualidade e Programa de Apoio à Capacitação Tecnológica, a Política Nacional de Informática a partir de 1992, o Programa de Competitividade Industrial (PCI) que, entre outros programas, não ocorreu efetivamente.

De qualquer forma, a Política Industrial brasileira, adotada nesse período, favoreceu tanto à modernização como o aumento do poder competitivo do parque industrial brasileiro, atingindo: ganhos de produtividade; significativas economias de capital de giro; estruturas administrativas e organizacionais enxutas e processos gerenciais mais eficientes. Em verdade, fruto da capacidade de resposta defensiva em função da "abertura comercial com instabilidade” demonstrada pelas empresas (Coutinho, 1994). 
Apesar do acentuado crescimento da produtividade da mão-de-obra, segundo Sabóia e Carvalho (1997), a produção industrial, a partir da década de 1990, não imprimiu uma dinâmica necessária a economia como um todo. Além disso, a estrutura produtiva brasileira tem uma participação pouco expressiva na produção de bens de capital, indústria esta, que mais dissemina tecnologia para outros setores Carleal (1997). Entretanto, de qualquer forma, atualmente, conforme De Negri e Salerno (2005), a indústria brasileira é uma das maiores e mais diversificadas dos países em desenvolvimento.

Com o novo Governo do início do século XXI, é implantada a Política Industrial, Tecnológica e de Comércio Exterior (PITCE) que é um marco recente no Brasil, pois, o governo integra as políticas: industrial, comércio exterior e de inovação tecnológica. Essa política é contemporânea, voltada para o futuro e busca induzir as mudanças necessárias no patamar competitivo da indústria brasileira.

A preocupação do Governo Lula envolveu a criação da Agência Brasileira de Desenvolvimento Industrial (ABDI) e do Conselho Nacional de Desenvolvimento Industrial (CNDI). Além da Lei de Inovação, que regula e facilita a relação entre instituições públicas e empresas privadas, e a Lei de Biossegurança, com propósitos entre outros de aumentar a competitividade da indústria nacional.

No entanto há de se ressaltar, que a competitividade necessária para participação no mercado internacional requer um forte investimento das economias periféricas em desenvolvimentos tecnológicos ligados a segmentos estratégicos como à microeletrônica, à biotecnologia e novos materiais.

\section{Dinâmica produtiva e a estrutura de ciência, tecnologia e inovação na Região Norte}

Conforme a literatura corrente e os diversos órgãos federais, Instituto Brasileiro de Geografia e Estatística (IBGE), Conselho Nacional de Desenvolvimento Científico e Tecnológico (CNPq), Instituto de Pesquisa Econômica e Social (IPEA), que mostram explicitamente os dados da Região Norte (alguns citados abaixo), se pode dizer que os estados do Pará e do Amazonas, são os que apresentam maior significado econômico na região. Dessa forma, faz-se necessário uma rápida contextualização das atividades produtivas atuais desses estados visando declarar a importância da região no âmbito nacional.

O estado do Pará, embora, nos últimos anos tenha experimentado uma ligeira diversificação e verticalização produtiva, atingindo tanto do setor agropecuário, quanto à indústria de transformação, ainda carrega uma forte dependência na exploração de recursos naturais e, em particular de recursos minerais (Diniz et al., 2007). Pesa, ainda, sobre a economia do estado do Pará 
uma grande dependência de atividades extensivas caracterizadas pela relativa intensidade no uso dos recursos naturais e trabalho como fatores produtivos principais, mas com baixa capacidade de agregação de valor.

A dependência das atividades econômicas de base mineral se projeta ainda mais para o futuro, uma vez que os investimentos no setor minério-metalúrgico no período 2005-2008, seja para a expansão da capacidade, ou para implantação de novas empresas, é estimado, segundo Enriquez (2003), em US $\$ 5,2$ bilhão para a indústria extrativa mineral e US\$2,6 bilhão para a indústria de transformação, com a geração esperada de 15.900 novos postos de trabalho.

No estado do Amazonas, por sua vez, o Polo Industrial de Manaus oferece uma dinâmica importante ao estado como um todo. A partir da década de 1990 até os dias de hoje, o Pólo Industrial de Manaus inserido no Modelo Zona Franca de Manaus, está ligada a uma fase de modernização industrial num ambiente, agora, globalizado (Almeida, 2008). Em função da abertura comercial brasileira, as indústrias incentivadas de Manaus, viram-se obrigadas a alterar substancialmente sua função de produção, passando a serem mais intensivas em capital e tecnologia, no qual passou a promover um esforço de modernização cujas velocidades e profundidade dificilmente possuem paralelo na história industrial brasileira (Machado et al., 2006).

\subsection{Dinâmica atual da produção econômica na Região Norte}

A despeito de cobrir $57 \%$ do território nacional, quando se analisa a participação da região no PIB nacional, observas-se que a mesma ainda é pouca expressiva. Em 2008, a região apresentou um PIB total de $\mathbf{R} \$ 133,5$ bilhões, correspondendo a somente $5,2 \%$ do PIB brasileiro, o que representou um pequeno aumentou quando comparado com o ano de 1998 quando essa participação era de 4,2\%. Quando se analisa o desempenho dos estados observa-se que o melhor desempenho foi do Pará cujo PIB correspondeu a $\mathrm{R} \$ 49$ bilhões, equivalendo a 1,8\% do total nacional. Roraima foi o estado da Região Norte que apresentou o menor PIB, R $\$$ 4,1 bilhões, ou seja, o,16\% do PIB nacional, conforme a Tabela 1. 
TABELA 1. VALOR DO PIB, PARTICIPAÇÃO DO PIB E PIB PER CAPITA, BRASIL - 1998 E 2008

\begin{tabular}{l|rrrr|r|r|r}
\hline \multirow{2}{*}{ Estados } & \multicolumn{4}{|c|}{1998} & \multicolumn{3}{c}{2008} \\
\cline { 2 - 7 } & \multicolumn{1}{c|}{ PIB } & \% PIB & PIB per c. & \multicolumn{1}{c|}{ PIB } & \% PIB & PIB per c. \\
\hline Acre & 1.694 & 0,17 & $3.181,81$ & 6.370 & 0,22 & 9.896 \\
Amapá & 2.038 & 0,21 & $4.596,50$ & 6.765 & 0,22 & 11.032 \\
Amazonas & 13.199 & 1,35 & $4.914,53$ & 46.823 & 1,54 & 14.014 \\
Pará & 15.091 & 1,54 & $2.527,89$ & 58.519 & 1,93 & 7.992 \\
Rondônia & 4.799 & 0,49 & $3.593,03$ & 17.888 & 0,59 & 11.976 \\
\hline Roraima & 1.347 & 0,14 & $4.412,44$ & 4.889 & 0,16 & 11.844 \\
Tocantins & 2.786 & 0,28 & $2.499,60$ & 13.091 & 0,43 & 10.223 \\
Norte & 40.954 & 4,18 & $3.446,79$ & 154.704 & 5,10 & 10.216 \\
Brasil & 979.276 & 100 & $5.910,38$ & 3.031 .864 & 100 & 15.989 \\
\hline
\end{tabular}

FONTE: IBGE, 2010.

Outro importante indicador, o PIB per capita, passou de uma média de R \$ 3,4 mil em 1998 para R \$ 10,2 mil em 2008, denotando um expressivo aumento da região, nesse período, no entanto, abaixo do PIB per capita nacional que passou de $\mathrm{R}$ \$ 5,9 mil para $\mathrm{R}$ \$ 15,9 mil no mesmo período analisado. Entre os estados, somente o Amazonas se aproximou da média nacional com R\$ 14,4 mil, enquanto o Pará apresentou o menor PIB per capita da região com R $\$ 7,9$ mil.

No que se refere a análise da participação das atividades produtivas conforme o valor adicionado bruto (VAB), pode-se observar, conforme demonstra a Tabela 02, que os estados do Amazonas e Pará se destacam em relação aos demais estados, em especial, no que diz respeito à atividade da indústria de transformação, onde no Amazonas essa indústria representou 30,7\% do valor adicionado bruto em 2008, e no Pará 10,8\%. Outras atividades preponderantes no Amazonas dizem respeito à administração, saúde e educação pública $(17,8 \%)$ e a atividade de comércio e serviços de manutenção e reparação $(11,5 \%)$, enquanto no Pará, se sobressaem ainda atividade de administração, saúde e educação públicas (17,7\%) e indústria extrativista com (14,0\%).

Ainda, conforme a Tabela 2, a participação da indústria de transformação na região Norte foi somente de 4,5\% enquanto que no âmbito nacional essa indústria participou com 16,6\%, sendo que somente o estado do Amazonas ficou a cima dessa média nacional. A atividade econômica mais importante da região no ano de 2008 foi a da Pecuária e Pesca com participação de $13,6 \%$, enquanto a indústria extrativa participou com 10,2\%, sendo a segunda atividade mais importante. Esses dados reforçam a predominância das atividades primárias sobre as atividades industriais, o que tem como conseqüência uma maior pressão sobre os recursos naturais e por conseguinte ao meio ambiente. 
TABELA 2. PARTICIPAÇÃO DAS ATIVIDADES ECONÔMICAS NO VALOR ADICIONADO BRUTO, SEGUNDO ESTADOS QUE COMPÕE A REGIÃO NORTE, BRASIL - 2008

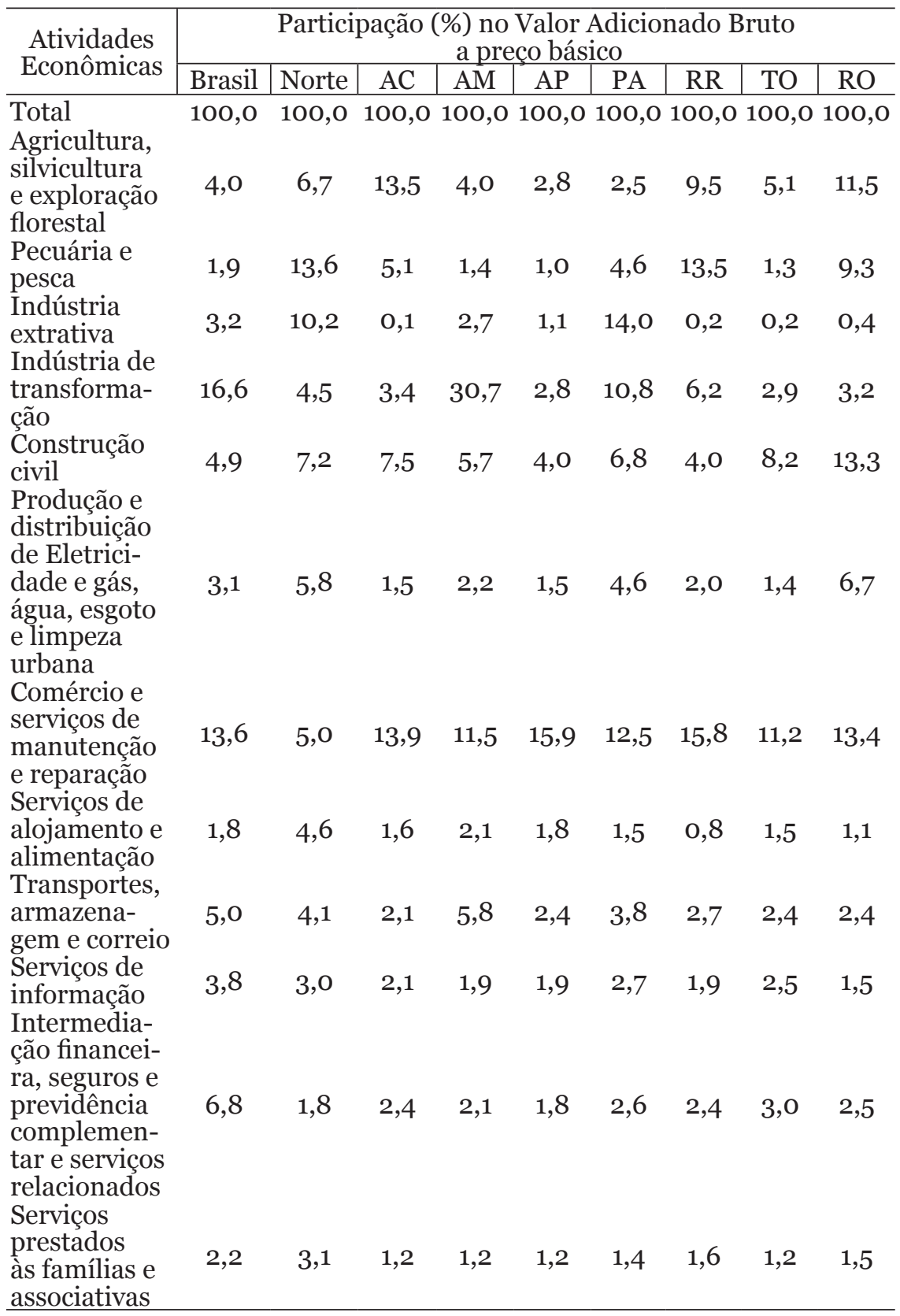


TABELA 2 (continuação). PARTICIPAÇÃO DAS ATIVIDADES ECONÔMICAS NO VALOR ADICIONADO BRUTO, SEGUNDO ESTADOS QUE COMPÕE A REGIÃO NORTE, BRASIL - 2008

\begin{tabular}{|c|c|c|c|c|c|c|c|c|c|}
\hline \multirow{2}{*}{$\begin{array}{l}\text { Atividades } \\
\text { Econômicas }\end{array}$} & \multicolumn{9}{|c|}{$\begin{array}{c}\text { Participação (\%) no Valor Adicionado Bruto } \\
\text { a preço básico }\end{array}$} \\
\hline & Brasil & Norte & $\mathrm{AC}$ & $\mathrm{AM}$ & $\mathrm{AP}$ & $\mathrm{PA}$ & RR & TO & RO \\
\hline $\begin{array}{l}\text { Serviços } \\
\text { prestados às } \\
\text { empresas }\end{array}$ & 4,9 & 2,3 & 1,9 & 2,7 & 1,6 & 2,1 & 1,7 & 3,0 & 1,5 \\
\hline $\begin{array}{l}\text { Atividades } \\
\text { imobiliárias e } \\
\text { aluguéis }\end{array}$ & 8,2 & 5,1 & 8,6 & 5,6 & 10,8 & 9,9 & 7,7 & 7,3 & 4,9 \\
\hline $\begin{array}{l}\text { Administra- } \\
\text { ção, saúde } \\
\text { e educação } \\
\text { pública }\end{array}$ & 15,8 & 7,5 & 33,4 & 17,8 & 46,2 & 17,7 & 27,9 & 47,3 & 23,8 \\
\hline $\begin{array}{l}\text { Saúde e } \\
\text { educação } \\
\text { mercantis }\end{array}$ & 3,0 & 2,6 & 0,8 & 1,8 & 1,9 & 1,3 & 1,2 & 0,7 & 1,9 \\
\hline $\begin{array}{l}\text { Serviços } \\
\text { domésticos }\end{array}$ & 1,2 & 4,7 & 1,1 & 0,7 & 1,3 & 1,3 & 1,0 & 0,7 & 1,3 \\
\hline
\end{tabular}

FONTE: IBGE, 2010.

\subsection{Os padrões atuais da ciência, tecnologia e inovação no Norte}

Uma forma de analisar o SRI de uma determinada região, e nesse caso, a região Norte e, portanto, o ritmo de crescimento econômico e social é a construção de um Índice Geral de Ciência, Tecnologia e Inovação (CT\&I). Isso se dá em função dos vários indicadores utilizados para sua elaboração, tais como: Gasto Per Capita Governamental; Percentual de Gasto em C\&T; Patentes; Taxa de Escolaridade de Jovens; Número de Pesquisadores por Milhão de Habitantes; Incubadoras de Empresas; Exportação de Produtos Intensivos em Tecnologia e outros ${ }^{6}$. Vale ressaltar que esse índice é relativo aos estados da região analisada. De forma alguma, está se fazendo alguma citação que o valor expressivo de 1 (um) é a melhor posição contando com os outros estados da federação.

A metodologia que foi utilizada para o cálculo dos Índices referese ao Technology Achievement Index (TAI) elaborado pelo United Nations Development Programme (UNDP), e divulgado no Relatório de Desenvolvimento Humano de 2001, que se tornou uma referência metodológica para a elaboração de índices dessa natureza.

6 Para melhor entendimento dos indicadores, da formulação dos índices e dos demais indicadores que não entraram nessa pesquisa ver ROCHA et al (2004). 
Segundo Diniz et al. (2009) a evidência empírica mais significativa do Índice de C,T\&I calculado para a região foi a sua fraca evolução no período analisado - 2000 a 2007, a exceção do estado do Amazonas que apresentou uma melhor posição relativa, conforme demonstra o Gráfico 1.

No caso do estado do Amazonas, como falado acima, essa posição relativa do índice se deve a dinâmica do Polo Industrial de Manaus (PIM). Segundo Diniz (2008) pode-se constatar que 68,06\% das empresas do PIM (numa amostra de 327 empresas, através de uma pesquisa de campo) inovam em produtos para o mercado local, nacional e com menor expressão para o mercado externo.

GRÁFICO 1. EVOLUÇÃO DO ÍNDICE GERAL DE CIÊNCIA E TECNOLOGIA DOS ESTADOS DA REGIÃO NORTE, BRASIL - 2000-2007

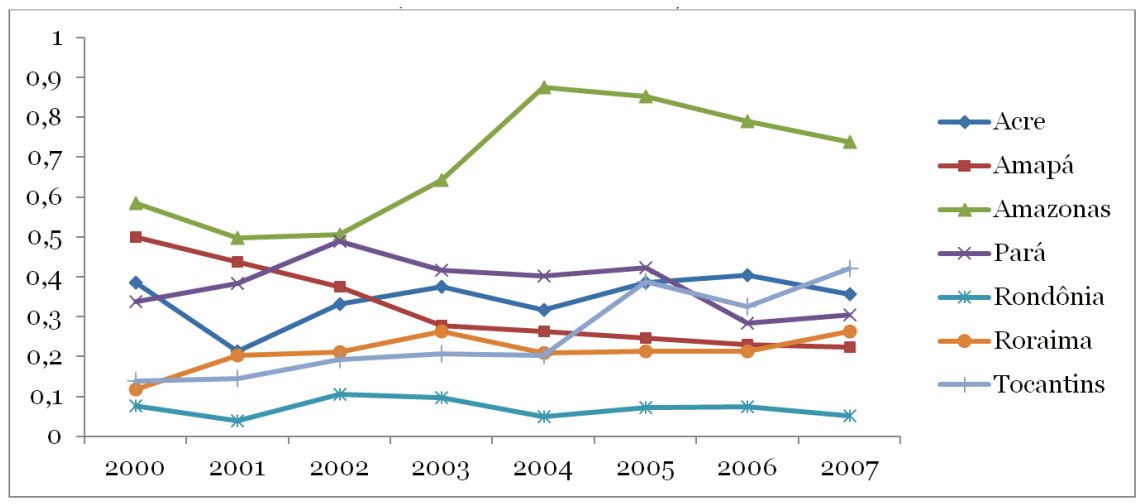

FONTE: Diniz et al., 2009.

O Estado do Pará apresenta uma posição decrescente em quase toda a série do índice geral e CT\&I. Sua pequena melhora se dá entre 2006 e 2007. Isso se deve à preocupação do governo estadual para o fomento a pesquisa, ciência e tecnologia, em especial, com a criação da Fundação de Amparo a Pesquisa do Estado do Pará (FAPESPA).

Os estados de Rondônia, Roraima, e Tocantins, como se esperava, apresentaram posição relativa irrelevante, conforme o Gráfico 1. 


\subsubsection{Uma análise a partir dos Grupos de Pesquisa}

Outro importante indicador de ciência e tecnologia é indicado na formação da estrutura cientifica coletado através dos grupos de pesquisas localizados nas instituições de ensino superior da Região. Alguns Estados destacam-se pelo maior número de grupos de pesquisa, tendo em vista o maior volume de linhas de pesquisa, pesquisadores, estudantes e técnicos existentes.

O estado do Pará lidera o ranking entre os estados da região com maior número de grupos de pesquisa, possuindo 379 grupos. Em seguida o Estado do Amazonas com 362 e Tocantins com 135, segundo a Tabela 3. No entanto, quando analisamos os itens linha de pesquisa, pesquisadores, estudantes e técnicos, o Estado do Amazonas ultrapassa todos os Estados, com um arcabouço de pesquisas mais diversificado.

Por outro lado, quando confrontamos os dados da região com os dados registrados no Brasil, constata-se um distanciamento das variáveis apresentadas. A maior participação corresponde ao número de pesquisadores, correspondente a $7,2 \%$ do total nacional e a menor equivale a $5,8 \%$ de estudantes na participação do total do país.

Apesar disso, Segundo Puty et al. (2008), já existem avanços na pesquisa cientifica, especialmente, através dos tipos de relacionamentos mais freqüentes entre grupos de pesquisas que interagem com empresas. Estes grupos de pesquisas interativos também se situam em maior número no Estado do Pará, representando 33,8\% do total da Região, que interagem com 68 empresas dentro e fora da região, conforme a Tabela 4.

Notadamente, o indicador de avanço científico identificado pelos tipos de relacionamento dos grupos com empresas tem confirmado (em estágio insipiente), o desenvolvimento de atividades na Região mais relacionadas à pesquisa cientifica, apresentando também transferência de tecnologia desenvolvida pelo grupo para empresas expresso pela Relação de número 7 da Tabela 4.

Este indicador revela que a pesquisa cientifica tem avançado lentamente, haja vista que as relações predominantes ainda são de caráter temporal reduzido, como expresso pela Relação 2, isto é, pesquisa científica com considerações de uso imediato dos resultados. No entanto, a Região Norte passa por transformações nas áreas produtivas que tem estimulado pesquisas mais aplicadas para atender a histórica deficiência em inovação tecnológica. 
ALMEIDA, L. M. de. et al. A importância das universidades e institutos de pesquisa...

TABELA 3. NÚMERO DE GRUPOS, PESQUISADORES, ESTUDANTES, TÉCNICOS, LINHAS DE PESQUISA E RELAÇÕES SEGUNDO UNIDADES DE FEDERAÇÃO, BRASIL - 2008

\begin{tabular}{lcccccc}
\hline \multicolumn{1}{c|}{ UF } & Grupos & $\begin{array}{c}\text { Linhas de } \\
\text { pesquisa }\end{array}$ & Pesquisadores & Estudantes & Técnicos \\
\hline Acre & 38 & 203 & 313 & 180 & 64 \\
Amapá & 36 & 117 & 206 & 142 & 36 \\
Amazonas & 362 & 1.664 & 3.362 & 3.028 & 682 \\
Pará & 379 & 1.492 & 2.892 & 2.650 & 485 \\
Rondônia & 48 & 202 & 390 & 303 & 25 \\
Roraima & 72 & 227 & 418 & 199 & 31 \\
Tocantins & 135 & 555 & 908 & 807 & 120 \\
Brasil & 22.797 & 86.075 & 159.948 & 177.702 & 24.143 \\
Norte & 1.523 & 6.126 & 11.582 & 10.275 & 1.724 \\
\hline
\end{tabular}

NOTA: Em geral há dupla contagem no número de pesquisadores, estudantes e técnicos, tendo em vista que o indivíduo que participa de mais de um grupo de pesquisa foi computado mais de uma vez.

FONTE: CNPq, Censo 2008.

TABELA 4. TIPO DE RELACIONAMENTO DO GRUPO SEGUNDO ESTADOS, REGIÕES E BRASIL - 2008

\begin{tabular}{|c|c|c|c|c|c|c|c|c|c|}
\hline Interações & $\mathrm{AC}$ & AP & $\mathrm{AM}$ & $\mathrm{PA}$ & $\mathrm{RO}$ & RR & TO & $\mathrm{NO}$ & $\mathrm{BR}$ \\
\hline Grupos & 1 & 6 & 33 & 52 & 8 & 4 & 13 & 154 & 2.726 \\
\hline Empresas & 8 & 8 & 45 & 68 & 12 & 4 & 25 & 228 & 3.865 \\
\hline Rel. 1 & $\mathrm{O}$ & 3 & 18 & 39 & 4 & 1 & 8 & 100 & 1.488 \\
\hline Rel. 2 & 5 & 4 & 23 & 47 & 3 & 4 & 9 & 121 & 3.061 \\
\hline Rel.3 & $\mathrm{O}$ & $\mathrm{O}$ & 5 & 12 & $\mathrm{O}$ & $\mathrm{O}$ & $\mathrm{O}$ & 20 & 334 \\
\hline Rel.4 & $\mathrm{O}$ & $\mathrm{O}$ & 1 & $\mathrm{O}$ & $\mathrm{O}$ & $\mathrm{O}$ & $\mathrm{O}$ & 2 & 130 \\
\hline Rel.5 & O & $\mathrm{O}$ & 1 & 2 & $\mathrm{O}$ & $\mathrm{O}$ & $\mathrm{O}$ & 4 & 119 \\
\hline Rel.6 & $\mathrm{O}$ & $\mathrm{O}$ & 2 & 7 & $\mathrm{O}$ & $\mathrm{O}$ & $\mathrm{O}$ & 10 & 281 \\
\hline Rel. 7 & 3 & 1 & 13 & 29 & 3 & 2 & 5 & 71 & 1.710 \\
\hline Rel.8 & $\mathrm{O}$ & 1 & 1 & 6 & 2 & $\mathrm{O}$ & 2 & 15 & 289 \\
\hline Rel.9 & $\mathrm{O}$ & $\mathrm{O}$ & 3 & 15 & 3 & $\mathrm{O}$ & 5 & 35 & 776 \\
\hline Rel. 10 & $\mathrm{O}$ & 1 & 6 & 12 & $\mathrm{O}$ & 1 & 6 & 35 & 665 \\
\hline Rel. 11 & O & 1 & 1 & 1 & 1 & O & 1 & 6 & 64 \\
\hline Rel. 12 & 3 & $\mathrm{O}$ & 7 & 15 & 1 & $\mathrm{O}$ & 3 & 44 & 612 \\
\hline Rel. 13 & $\mathrm{O}$ & $\mathrm{O}$ & 3 & 7 & $\mathrm{O}$ & $\mathrm{O}$ & $\mathrm{O}$ & 13 & 196 \\
\hline Rel. 14 & $\mathrm{O}$ & 2 & 2 & 3 & 4 & $\mathrm{O}$ & 4 & 20 & 698 \\
\hline
\end{tabular}

NOTA: As empresas não estão localizadas, necessariamente, na mesma área geográfica do grupo; Não há dupla contagem no número de empresas na dimensão mais desagregada da tabela, mas há dupla contagem nos totais obtidos por soma. Isto porque a mesma empresa pode ser mencionada por grupos localizados em mais de uma UF ou Região; Foram admitidos até três tipos de relacionamento.

FONTE: CNPq, Censo 2008. 
O Quadro 1 apresenta os tipos de relacionamentos entre os grupos de pesquisas interativos com empresas, considerados pelo CNPq.

QUADRO 1. TIPOS DE RELACIONAMENTOS ENTRE OS GRUPOS DE PESQUISA, BRASIL - 2008

\begin{tabular}{|l|}
\hline \multicolumn{1}{|c|}{ Legenda para Tipo de Relacionamento } \\
\hline Rel1 - Pesquisa científica sem considerações de uso imediato dos resultados \\
\hline Rel2 - Pesquisa científica com considerações de uso imediato dos resultados \\
\hline $\begin{array}{l}\text { Rel3 - Atividades de engenharia não-rotineira inclusive o desenvolvimento de } \\
\text { protótipo cabeça de série ou planta-piloto para o parceiro }\end{array}$ \\
\hline $\begin{array}{l}\text { Rel4 - Atividades de engenharia não-rotineira inclusive o desenvolvimento/fabri- } \\
\text { cação de equipamentos para o grupo }\end{array}$ \\
\hline Rel5 - Desenvolvimento de software não-rotineiro para o grupo pelo parceiro \\
\hline Rel6 - Desenvolvimento de software para o parceiro pelo grupo \\
\hline Rel7 - Transferência de tecnologia desenvolvida pelo grupo para o parceiro \\
\hline Rel8 - Transferência de tecnologia desenvolvida pelo parceiro para o grupo \\
\hline Rel9 - Atividades de consultoria técnica não contempladas nos demais tipos \\
\hline $\begin{array}{l}\text { Rel10 - Fornecimento, pelo parceiro, de insumos materiais para as atividades de } \\
\text { pesquisa do grupo sem vinculação a um projeto específico de interesse mútuo }\end{array}$ \\
\hline $\begin{array}{l}\text { Rel11 - Fornecimento, pelo grupo, de insumos materiais para as atividades do par- } \\
\text { ceiro sem vinculação a um projeto específico de interesse mútuo }\end{array}$ \\
\hline $\begin{array}{l}\text { Rel12 - Treinamento de pessoal do parceiro pelo grupo incluindo cursos e treina- } \\
\text { mento "em serviço" }\end{array}$ \\
\hline $\begin{array}{l}\text { Rel13 - Treinamento de pessoal do grupo pelo parceiro incluindo cursos e treina- } \\
\text { mento "em serviço" }\end{array}$ \\
\hline $\begin{array}{l}\text { Rel14 - Outros tipos predominantes de relacionamento que não se enquadrem em } \\
\text { nenhum dos anteriores }\end{array}$ \\
\hline
\end{tabular}

FONTE: CNPq, Censo 2008.

\section{Aspectos metodológicos}

O presente artigo trabalha com os resultados do Projeto de Pesquisa: interações de universidades e institutos de pesquisas com empresas no Brasil, coordenado por Suzigan e Albuquerque (2008), do qual os autores deste artigo fazem parte. A metodologia proposta, avaliar os dados dos grupos de pesquisa registrados no Diretório de Grupos de Pesquisa do CNPq. Essa instituição fornece em sua base de dados diversas informações sobre os grupos de pesquisa pertencentes à universidades e Institutos Públicos de Pesquisa (Hit), conforme metodologia proposta por Rapini (2006). Entre as informações, constam 
os grupos que realizaram algum tipo de interação com empresas do setor produtivo. A partir dessas informações, foi possível identificar 40 empresas que realizaram efetivamente interações com universidades e/ou IPPs nos sete Estados que compõe a Região Norte. Desse total, 19 empresas responderam ao questionário prestando informações avaliativas a respeito da interação com as universidades e/ou IPPs. A maioria dessas empresas está localizadas nos Estados do Pará e Amazonas com 7 e 6 empresas respectivamente ou seja aproximadamente $70 \%$ do total. No Amapá, 3 empresas responderam o questionário, em Rondônia 2 empresas e em Roraima somente 1 empresa. Tocantins e Acre foram os dois únicos estados que não constaram empresas interativas.

No que se refere ao setor ao qual se vinculam as empresas que compõe a amostra, a maioria, sete empresas ou (36,8\%), está associada à indústria de transformação, enquanto o setor de Eletricidade e Gás abrange cinco empresas ou $(26,3 \%)$ do total. As demais empresas estão representadas no setor de Pesquisa e Desenvolvimento com quatro empresas, setor de Agricultura, Pecuária, Silvicultura e Exploração Florestal com duas empresas e o setor Extrativista com uma empresa, conforme se observa na Tabela 5.

TABELA 5. NÚMERO DE EMPRESAS POR SETOR PRODUTIVO NA REGIÃO NORTE, BRASIL - 2009

\begin{tabular}{l|cc}
\hline \multicolumn{1}{c|}{ Setores } & No Empresas & (\%) \\
\hline Agricultura, Pecuária, Silvicultura e Exploração Florestal & 2 & 10,5 \\
Indústria Extrativa & 1 & 5,3 \\
Indústria de Transformação & 7 & 36,8 \\
Eletricidade e Gás & 5 & 26,3 \\
Pesquisa e Desenvolvimento Científico & 4 & 21,1 \\
Total & 19 & 100,0 \\
\hline
\end{tabular}

FONTE: Pesquisa de Campo, 2009. 
Os dados extraídos do questionário aplicado junto às empresas foram tabulados segundo a metodologia utilizada pela Rede de Sistemas Produtivo e Inovativos Locais (REDESIST) ${ }^{7}$, que permite ponderar a percepção de importância dos respondentes que é uma variável qualitativa em uma variável quantitativa ${ }^{8}$. Se obtendo os índices de grau de importância quantitativos que seguem uma escala de valores entre (o e 1). Nessa perspectiva, quanto mais próximo de um, mais a empresa percebe quão importante é determinada atividade; do mesmo modo, quanto mais próximo de zero menos importante a atividade.

\section{Dinâmica da Interação Universidades e IPPs com Empresas na Região}

As atividades inovadoras compreendem todas as etapas necessárias para o desenvolvimento de produtos (inovações radicais) ou processos (inovações incrementais) novos ou melhorados, que possibilitam a ampliação dos conhecimentos das empresas para produzir e comercializar bens e serviços conforme abordado por Kim e Nelson (2005). Logo, na presente pesquisa, foram identificados quais os tipos de atividades inovadoras que são desenvolvidas pelas empresas em parceria com universidades e institutos públicos de pesquisa (IPPs), analisando as estratégias utilizadas, a importância das universidades e IPPs, a relevância das áreas do conhecimento para o setor produtivo da região, além das principais razões que levaram à interação.

No que se refere aos tipos de inovações realizadas pelas empresas, que podem ser de produto ou processo produtivo, os resultados observados na Tabela 6 , mostram que somente $15,8 \%$ não realizou nem um tipo de inovação em produtos, enquanto que $5,3 \%$ das empresas responderam ter desenvolvido um produto novo para o mundo, 26,3\% afirmaram ter desenvolvido um produto novo para o mercado nacional, $42,1 \%$ afirmaram ter desenvolvido um produto novo para a empresa e 52,6\% afirmaram ter realizado somente aperfeiçoamento de um produto já existente. Por outro lado, as inovações em processo são mais comuns para estas empresas na região, onde 73,7\% responderam ter realizado aperfeiçoamento de um processo já existente, $21,1 \%$ desenvolveram um processo novo para a empresa, $15,8 \%$ um processo novo para o país.

7 A Rede de Sistemas Produtivos e Inovativos Locais - RedeSist é uma rede de pesquisa interdisciplinar, formalizada desde 1997, sediada no Instituto de Economia da Universidade Federal do Rio de Janeiro e que conta com a participação de várias universidades e institutos de pesquisa no Brasil, além de manter parcerias com outras instituições do exterior (Lastres; Cassiolato, 2003).

8 Índice $=\left(\mathrm{N}^{\mathrm{o}}\right.$. sem importância* $\mathrm{O}+\mathrm{N}^{\mathrm{o}}$. pouco importante $\mathrm{O}, 3+\mathrm{N}^{\mathrm{o}}$. moderadamente importante $\mathrm{O}, 6 \mathrm{e}$ $\mathrm{N}^{0}$. muito importante* 1,0$) /\left(\mathrm{N}^{\circ}\right.$. respostas questionário). 
Somente $5,3 \%$ responderam que não realizaram nem um processo novo. Esses resultados chamam a atenção para a importância da inovação de produtos que apesar de incipiente, começa a ganhar relevância na medida em que aumentam os incentivos públicos a C,T\&I na região.

TABELA 6. INOVAÇÕES REALIZADAS PELAS EMPRESAS, BRASIL - 2009

\begin{tabular}{lc}
\multicolumn{1}{c}{ Tipos de Inovação } & Empresas (\%) \\
\hline Produto & \\
Nenhum produto novo & $15,8 \%$ \\
Aperfeiçoamento de um produto já existente & $52,6 \%$ \\
Novo para a empresa, mas não para o país & $42,1 \%$ \\
Novo para o país, mas não para o mundo & $26,3 \%$ \\
Novo para o mundo & $5,3 \%$ \\
\hline Processo & \\
Nenhum processo novo & $5,3 \%$ \\
Aperfeiçoamento de um processo já existente & $73,7 \%$ \\
Novo para a empresa, mas não para o país & $21,1 \%$ \\
Novo para o país, mas não para o mundo & $15,8 \%$ \\
Novo para o mundo & $0 \%$ \\
\hline
\end{tabular}

FONTE: Pesquisa de Campo, 2009.

\subsection{Fontes de Informação}

No presente estudo, foram avaliadas as fontes de informação prestadas pelas universidades e IPPs. No que se refere às fontes de informações geradas pelas universidades para a realização de atividades inovativas das empresas, os dados apresentados no Gráfico 2 consideram os principais fatores segundo o seu grau de importância para as empresas da região analisada.

Conforme se observa, os três principais fatores de informação para as empresas na região Norte, são as publicações e relatórios, com indicador de importância de 0,69 , em seguida, são consideradas as pesquisas encomendadas à universidades e pessoal contratado com graduação ou pós, ambos com os mesmo indicador de importância, isto é o,66. As variáveis pesquisas realizadas em conjunto e conferências públicas e encontros, também apresentaram relevância quanto as fontes de informação disponibilizadas pelas universidades na região Norte. Do mesmo modo, as empresas consideraram como sendo de pouca importância, as variáveis intercâmbio temporário de pessoal, com indicador de 0,34 , tecnologia licenciada com o,35 e por último Incubadoras com 0,25 . 
Considerando que as fontes de informação oriundas de agentes externos a empresa, como no caso das instituições analisadas, requerem que a mesma possua significativa capacitação tecnológica para poder adquirir, assimilar e aperfeiçoar o conhecimento obtido fora de suas instalações, pode-se observar que as universidades cumprem um importante papel para complementar e até mesmo substituir pesquisas dessas empresas na região.

GRÁFICO 2. IMPORTÂNCIA DAS FONTES DE INFORMAÇÃO DE UNIVERSIDADES NA REGIÃO NORTE, BRASIL - 2009

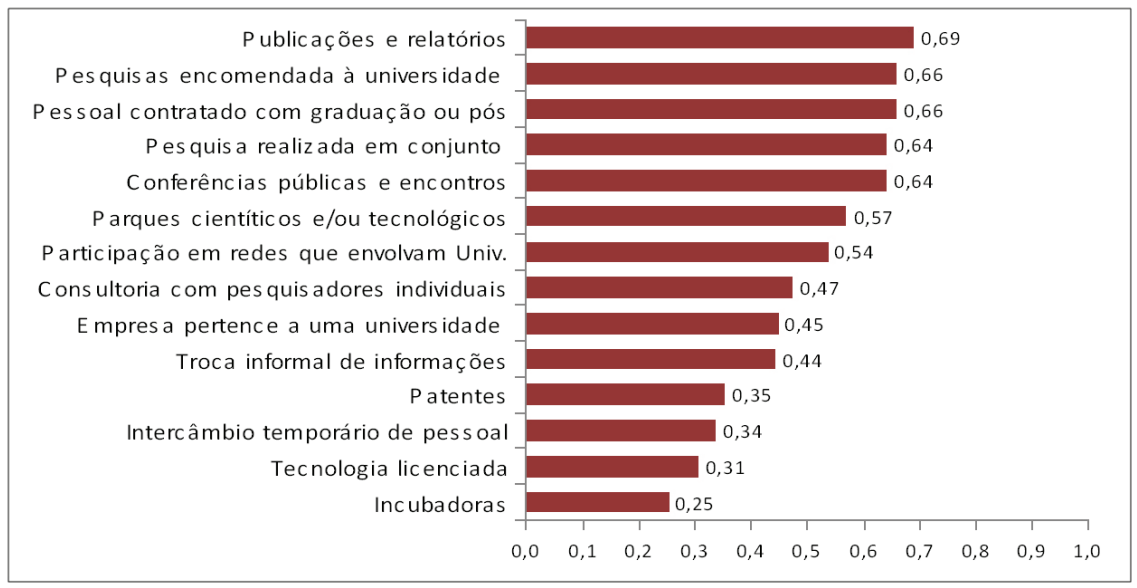

FONTE: Pesquisa de Campo, 2009.

Quando se analisa as fontes de informação prestadas pelos IPPs, observase, conforme demonstra o Gráfico 3, que os fatores mais relevantes para as empresas, dizem respeito à pesquisas realizadas em conjunto, o qual apresentou indicador de importância de 0,66 , enquanto que pesquisas encomendadas junto aos IPPs e conferências públicas aparecem em segundo lugar empatados com indicador de 0,59. De outro modo, os fatores patentes, com indicador de 0,37, tecnologia licenciada, com indicador de 0,34, e incubadoras com 0,26, se apresentaram como os de menor relevância para as empresas. No caso dos IPPs, também pode ser observado a relevância da interação dessas instituições com as empresas, mesmo que numa intensidade um pouco menor que com as universidades. 
GRÁFICO 3. IMPORTÂNCIA DAS FONTES DE INFORMAÇÃO DE IPPs NA REGIÃO NORTE, BRASIL - 2009

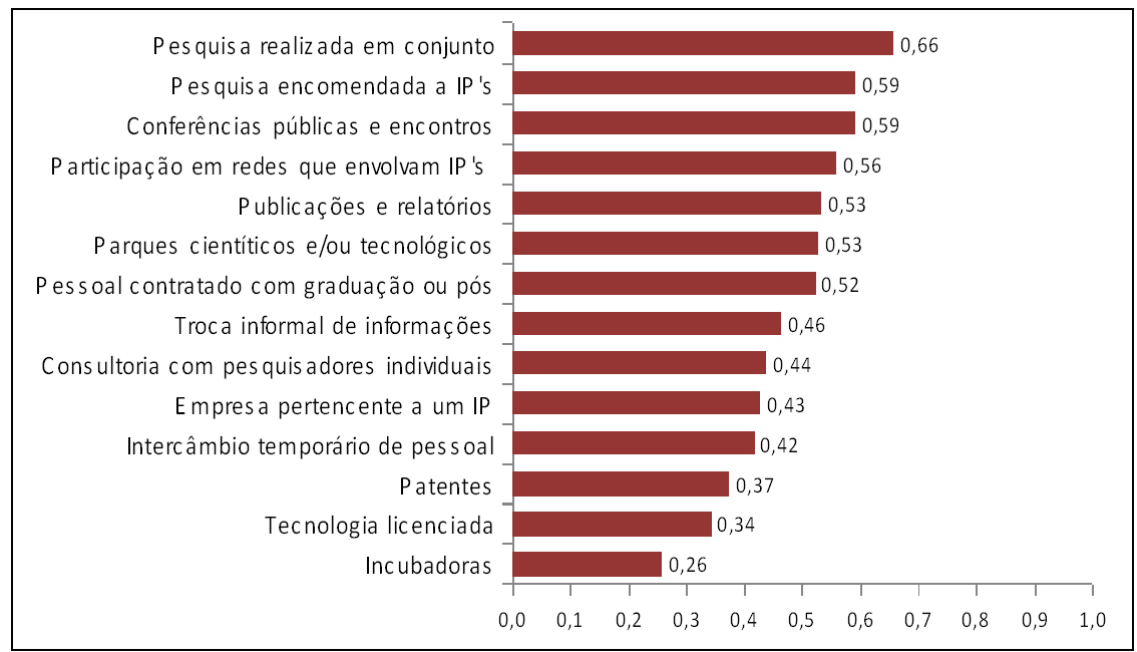

FONTE: Pesquisa de Campo, 2009.

\section{2 Área do conhecimento}

No que se refere às disciplinas científicas e de engenharias, segundo a classificação do $\mathrm{CNPq}$, a presente seção avalia as áreas do conhecimento que mais contribuíram para as atividades de P\&D e inovação das empresas.

O Gráfico 4 abaixo mostra o resultado sobre a relevância da pesquisa pública conduzida por universidades, distribuído nas seguintes disciplinas das áreas cientificas e de engenharia, e sua contribuição à inovação industrial.

Nos Estados da região Norte, os campos do conhecimento considerados mais importantes para as atividades produtivas das empresas foram: engenharia elétrica, que obteve grau de importância moderada com indicador de 0,49, em segundo ficou a área da ciência da computação, com indicador de 0,44 e em terceiro engenharia de minas com indicador de 0,43. Do lado oposto, as disciplinas com menor relevância segundo as empresas foram: desenho industrial, medicina e matemática. Conforme se pode observar, nenhuma das disciplinas apresentou grau de importância a cima de o,6 o que decorre da heterogeneidade produtiva das empresas que compõe a amostra, sobretudo as pertencentes aos dois Estados mais importantes economicamente da região, isto é, Pará e Amazonas. 
Destaca-se notadamente, os fluxos do conhecimento observados que oferecem ampla contribuição e possuem maior dinâmica na relação entre academia e setor produtivo, nesses Estados. No primeiro se sobressai as áreas de Engenharia Elétrica e Engenharia de Minas, que estão diretamente relacionadas as atividades de extração mineral, lideradas pela empresa Vale e pela atividade de eletricidade coordenado pela Eletronorte. Enquanto no Estado do Amazonas, a área da Engenharia da computação se sobressai, dado a importância do setor eletroeletrônico no Pólo Industrial do Estado, composto por empresas como a Samsung e Nokia por exemplo.

\section{GRÁFICO 4. IMPORTÂNCIA DAS ÁREAS DE CONHECIMENTO PARA AS} EMPRESAS, BRASIL - 2009

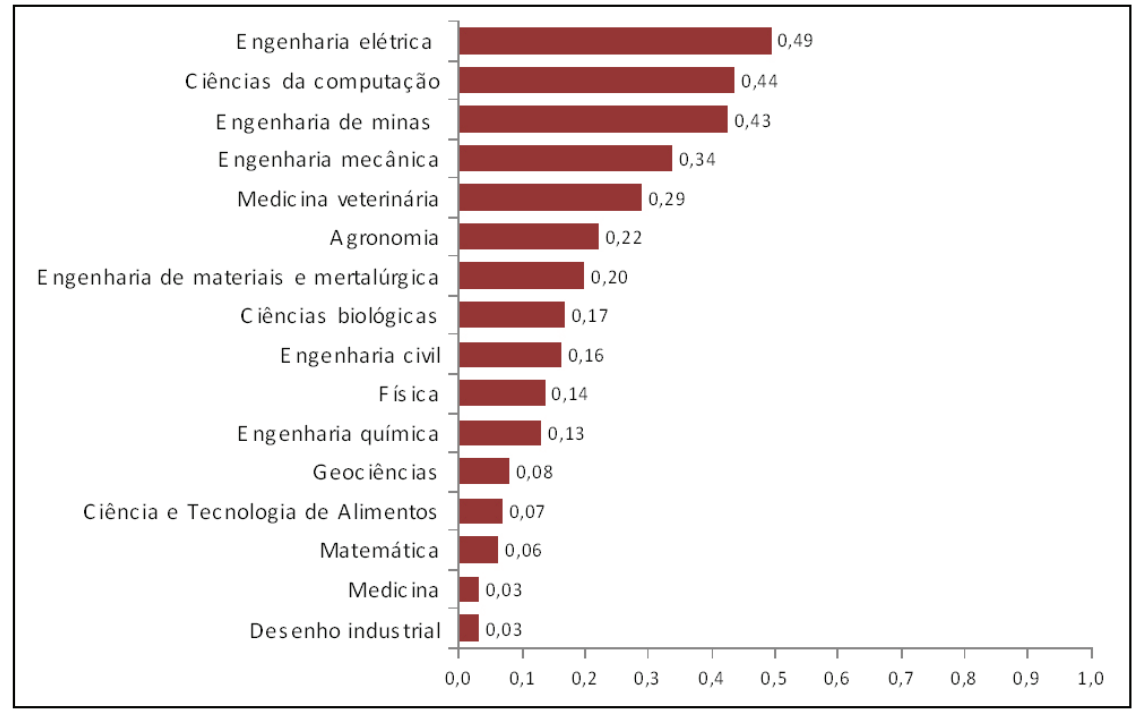

FONTE: Pesquisa de Campo, 2009.

Além disso, esses dados comprovam o destacado papel das áreas de engenharia, consideradas as áreas mais relevantes do meio acadêmico para o setor produtivo dado à dinâmica do mercado. Logo as áreas do conhecimento de maior dinamismo nesses estados, estão diretamente ligadas, aos setores produtivos potenciais da região. Constata-se assim que existem áreas do conhecimento que possuem maior propensão a gerar e transferir tecnologia do que outras, conforme aponta a literatura.

Cabe mencionar ainda a reduzida importância atribuída ás áreas voltadas para a biotecnologia, mesmo a região compreendendo a maior parte da Amazônia brasileira, isto é, o bioma que apresenta a maior biodiversidade do país. Esse fato demonstra um grave descompasso entre o verdadeiro potencial da região e a dinâmica do setor produtivo. 


\subsection{Resultado da pesquisa para a empresa}

Dentre os resultados das parcerias de pesquisa entre universidades e institutos públicos de pesquisa com empresas, podem ser considerados para o presente tópico, os resultados de pesquisa, os protótipos, as novas técnicas e instrumentos além dos laboratórios e metrologia.

O Gráfico 5 aponta à importância do resultado e recursos produzidos pelas universidades e IPPs para as atividades inovativas das empresas da região. Dos resultados observados, as contribuições das universidades e IPPs que representaram maior grau de importância foram: resultados da pesquisa com indicador de importância de 0,77 e as novas técnicas e instrumentos com indicador de importância o,68. Já os laboratórios apresentaram um indicador de importância intermediário, com índice de 0,56, assim como os resultados de protótipos com 0,51 .

GRÁFICO 5. IMPORTÂNCIA DOS RESULTADOS DAS UNIVERSIDADES E IPPS PARA AS EMPRESAS, BRASIL - 2009



FONTE: Pesquisa de Campo, 2009.

Deste modo, os dados reforçam a importância das universidades e o papel complementar e substituto da pesquisa para o P\&D da indústria, pois algumas empresas que realizam atividades de $P \& D$ vinculadas com universidades não possuem laboratórios de pesquisa, ou possuem departamento mais utilizam a estrutura das universidades para auxiliar suas atividades. 


\subsection{Razões da colaboração}

Quando se avalia as razões que levaram as empresas a colaborarem com universidades e/ou IPPs, observa-se, conforme demonstra o Gráfico 6, que a principal razão apontada diz respeito a aumentar a habilidade da empresa, cujo indicador de importância atribuído foi de o,63, enquanto que buscar conselhos de cunho tecnológico e contratar pesquisas complementares aparecem com igual indicador de importância, isto é o,55, ou seja, um grau de importância intermediário. Por outro lado, realizar testes necessários para produtos e processos e receber ajuda no controle de qualidade foram os fatores que receberam menor grau de importância, com indicador de 0,17 para ambos. $\mathrm{O}$ que demonstra a autonomia das empresas, quanto a estes elementos em relação as instituições analisadas.

\section{GRÁFICO 6. RAZÕES DA COLABORAĈ̃̃ COM UNIVERSIDADES E/OU} IPPs SEGUNDO O GRAU DE IMPORTÂNCIA, BRASIL - 2009

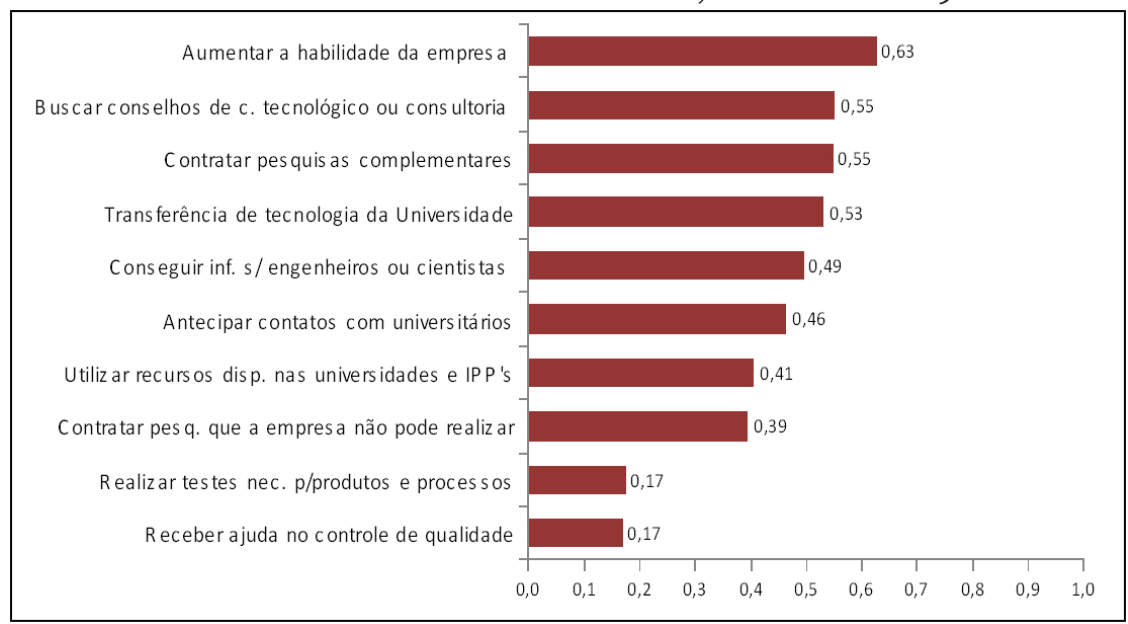

FONTE: Pesquisa de Campo, 2009. 


\section{Considerações Finais}

O presente artigo buscou analisar os padrões de interação existente entre universidades e IPPs com empresas na região Norte, visando compreender a importância dessas instituições para o desenvolvimento do Sistema de Inovação da Região.

Os resultados preliminares do estudo indicam que na região Norte, a interação entre universidades e IPPs com empresas possui um papel de destaque na medida em que as empresas que não exercem atividade de Pesquisa e Desenvolvimento (P\&D) interno absorvem o conhecimento cientifico produzido pelas universidades e IPPs. Esse fato é destacado nas respostas das empresas que apontam as pesquisas encomendadas por elas junto às instituições analisadas, como uma das fontes de informação mais relevantes para as atividades inovativas das empresas.

Os resultados também demonstram que algumas áreas do conhecimento científico, como Engenharia Elétrica, Ciências da Computação e Engenharia de Minas, são mais relevantes para o setor produtivo da região. O que se deve a dinâmica produtiva dos dois estados mais importantes economicamente, isto é Pará e Amazonas. No primeiro, prevalecendo às atividades de distribuição de energia e extração de minérios e no segundo a atividade do setor eletroeletrônico do Pólo Industrial de Manaus. Por outro lado, é notória a pouca participação de áreas voltadas para a biotecnologia na região, mesmo esta compreendendo a maior parte da Amazônia brasileira, que por sua vez compreende o bioma com a maior biodiversidade do país e um dos maiores do mundo. Esse fato requer um esforço maior da esfera pública no sentido de induzir atividades produtivas voltadas para esses setores.

Nesse aspecto, o grande desafio é compreender o relevante papel das universidades e IPPs para o sistema regional de inovação, incorporando-o como base das estratégias e políticas setoriais, voltadas para a promoção do desenvolvimento sustentável dessa região amazônica. O desafio das instituições e das empresas é reconhecer-se como parte desse sistema e promover a adequação dos mecanismos e instrumentos de execução das políticas setoriais aos fundamentos e às condições essenciais para a realização do mesmo.

Em termos da construção do sistema de inovação na Região, o problema reside, por um lado, na limitação desse núcleo de empresas, que restringe a propagação dos efeitos positivos derivados da interação com universidades e IPPs, o que se reforça pelo pequeno número de empresas que realizam investimentos em P\&D.

Por outro lado, é necessário reforçar o papel das universidades e institutos públicos de pesquisa. A pesquisa apontou que a infraestrutura científica está 
aquém de um patamar que indica o alcance de um conhecimento necessário para aumentar a eficiência da interação ciência e tecnologia. O crescimento da infraestrutura científica é necessário para que essas instituições consigam atender à demanda que venha a crescer em empresas mais envolvidas em inovação, o que vai resultar, também, no aumento da demanda por profissionais formados nas universidades. E isso é constatado através dos baixos indicadores que comparam o Índice de CT\&I para a região Norte, em especial, do Gasto Per Capita Governamental; Percentual de Gasto em C\&T; Taxa de Escolaridade de Jovens; Número de Pesquisadores por Milhão de Habitantes. O que requer esforços, sobretudo dos governos estaduais e Federal para fomentar tais elementos na região.

\section{Referências bibliográficas}

ALMEIDA, L. M. (2008). Inovações Tecnológicas e Interações Industriais: Um Estudo do Desenvolvimento Local a Partir da Cadeia Intermediária da Nokia no PIM. Belém: Dissertação de Mestrado. Universidade Federal do Pará, Programa de Pós Graduação em Economia.

ARROIO, A.; CASSIOLATO, J.; LASTRES, H. M. [org.] (2005). Conhecimento, Sistemas de Inovação e Desenvolvimento. Rio de Janeiro: Editora UFRJ.

ASHEIM, B.; COHENEN, L. (2005). "Knowledge Bases and Regional Innovation Systems: Comparing Nordic Clusters.” Research Policy, 34; p. 1173-1190.

CARLEIAL, L.; VALLE, R. [Org.] (1997). Reestruturação Produtiva e Mercado de Trabalho no Brasil. São Paulo: Hucitec.

CASSIOLATO, J. E.; LASTRES, H. M. M. (1998). "Local Systems of Innovation in the Mercosur of the 1990s: A Contribution to the Debate on S\&T Policy Decentralization." Workshop Techregiões: ciência, tecnologia e desenvolvimento.

COHEN, W. M.; NELSON, R. R.; WALSH, J. P. (2002). "The Influence of Public Research on Industrial R\&D.” Management Science, 48(1).

CONSELHO NACIONAL DE DESENVOLVIMENTO CIENTÍFICO E TECNOLÓGICO, 2005. URL [on-line]: www.cnpq.br. Acesso em: o5 de ago. de 2007.

COSTA, F.; MACHADO, J. A.; OLIVEIRA Jr, A.; SANTANA, A. (2006). "Metamorfoses do modelo Zona Franca de Manaus: desafios à pesquisa e ao planejamento do desenvolvimento regional." In: SCHERER, E.; OLIVEIRA, Jr. A. [orgs.]. Amazônia: políticas públicas e diversidade cultural. Rio de Janeiro: Garamond. 
COUTINHO, L. G. (1996). "A Fragilidade do Brasil em Face da Globalização.” In: BAUMANN, R. [org.]. O Brasil e a Economia Global. Rio de Janeiro: Campus.

COUTINHO, L. G. (1994). Superação da fragilidade tecnológica e a ausência de cooperação. Ciência e tecnologia: alicerces do desenvolvimento. São Paulo: Cobram.

DE NEGRI, J. A.; SALERNO, M. S. [org.]. (2005). Inovações, padrões tecnológicos e desempenho das firmas industriais brasileiras. IPEA.

DINIZ, M. B. et al. (2007). "A Amazônia (Legal) Brasileira: evidências de uma condição de armadilha da pobreza?” Anais do $35^{\circ}$ Encontro Nacional de Economia.

DINIZ, M. J. T. (2008). A Dinâmica das inovações nas empresas do pólo industrial de Manaus: um novo momento relacionado aos constrangimentos ambientais a partir do ano de 20oo. Tese de Doutorado. Universidade Federal do Pará (UFPA).

DINIZ, M. J. T. et al. (2009). "Evolução dos Índices de Ciência, Tecnologia e Inovação - ICT\&I para os Estados da Amazônia Legal no Período entre 2000 e 2007." Novos Cadernos do NAEA 248; p. 3-28.

DOSI, G. (2006). Mudança Técnica e Transformação Industrial: a teoria e uma aplicação a indústria de semicondutores. Campinas: Unicamp.

DOSI, G. (1984). "Technology and Conditions of Macroeconomic Development." In: FREEMAN, C. Design, Innovation and Long Cycles in Economic Development. New York: St. Martin's Press.

DOSI, G.; FREEMAN, C.; NELSON, R.; SILVERBERG, G.; SOETE, L. (1990). Technical change and economic theory. London: Pinter Publishers, 1990.

ENRÍQUEZ, G.; SILVA, M. A.; CABRAL, E. (2003). Biodiversidade da Amazônia: Usos e potencialidades dos mais importantes produtos naturais. Belém: NUMA/UFPA.

ENRÍQUEZ, M. A. S. (2008). Mineração - Maldição ou Dádiva? Os dilemas do desenvolvimento sustentável a partir de uma base mineira. São Paulo: Signus Editora.

INSTITUTO BRASILEIRO DE GEOGRAFIA E ESTATÍSTICA. (2005). URL [on-line]: www.ibge.gov.br. Acesso em: 12 de set. de 2007.

KLEVORICK, A. K.; LEVIN, R.; NELSON, R.; WINTER, S. (1995). “On the Sources and Significance of Interindustry Differences in Technological Opportunities." Research Policy, 24(2); p. 185-205. 
NELSON, R.; KIM, L. (2005). Tecnologia Aprendizado e Inovação: as experiências de economias de industrialização recente. Campinas: Editora da Unicamp.

NELSON, R. R.; WINTER, S. G. (1982). An evolutionary theory of economic change. Cambridge: Harvard University.

PUTY, C. A. B.; ARAUJO, L, M.; ALMEIDA, L. M. (2008). "Interações entre Universidades e Institutos de Pesquisa com Empresas na Amazônia." VI Encontro da Associação Brasileira de Estudos Regionais e Urbanos.

RAPINI, M. S.; RIGHI, H. (2006). "Metodologia para o Mapeamento da Interação Universidade-Empresa”. Diretório dos Grupos de Pesquisa do CNPq no Censo 2004.

ROCHA, E. M. P.; FERREIRA, M. A. T. (2004). "Indicadores de Ciência, Tecnologia e Inovação: mensuração dos Sistemas de CT\&I nos Estados Brasileiros." Ciência da Informação, 33(3).

ROSENBERG, N. (2006). Por dentro da caixa preta: Tecnologia e economia. Campinas: Editora da Unicamp.

SABÓIA, J.; CARVALHO, P. G. M. (1997). "Produtividade na Indústria Brasileira. Questões Metodológicas e Análise Empírica”. Texto para Discussão, $\mathrm{n}^{0}$ 504. Brasília: IPEA.

SUZIGAN, W.; ALBUQUERQUE, E. (2006). "Interações de universidades e institutos de pesquisa com empresas no Brasil." Projeto de Pesquisa: PCT/UNICAMP e Cedeplar/UFMG.

SUZIGAN, W.; ALBUQUERQUE, E. (2008). “A interação entre universidades e empresas em perspectiva histórica no Brasil.” Texto para discussão, 329. Belo Horizonte: Cedeplar/UFMG. 
ALMEIDA, L. M. de. et al. A importância das universidades e institutos de pesquisa... 